\title{
Germanica
}

\section{Ulrich Schacht. Identité paysagère, politique et poétique}

Ulrich Schacht. Landschaft als politische und poetische Identität

\section{Maryse Jacob}

\section{(2) OpenEdition}

\section{Journals}

Édition électronique

URL : http://journals.openedition.org/germanica/349

DOI : 10.4000/germanica.349

ISSN : 2107-0784

\section{Éditeur}

Université de Lille

\section{Édition imprimée}

Date de publication : 1 décembre 2006

Pagination : 215-227

ISBN : 2-913857-18-3

ISSN : 0984-2632

\section{Référence électronique}

Maryse Jacob, «Ulrich Schacht. Identité paysagère, politique et poétique », Germanica [En ligne], 39 |

2006, mis en ligne le 07 janvier 2010, consulté le 06 octobre 2020. URL : http://

journals.openedition.org/germanica/349; DOI : https://doi.org/10.4000/germanica.349

Ce document a été généré automatiquement le 6 octobre 2020.

(ㄷ) Tous droits réservés 


\title{
Ulrich Schacht. Identité paysagère, politique et poétique
}

\author{
Ulrich Schacht. Landschaft als politische und poetische Identität
}

\author{
Maryse Jacob
}

1 Le constat d'Hermann Korte au terme de son étude sur la poésie allemande depuis la césure de la Deuxième Guerre mondiale cerne sans détour la difficulté du lyrisme contemporain à s'engager dans des voies nouvelles, après l'usure de modes d'expression spécifiques grâce auxquels les générations précédentes avaient pu répondre à la crise de la représentation héritée du XIXe siècle et à l'effondrement de l'univers magique :

À la fin des années 80 , la poésie lyrique ne se fie plus à aucune orientation
susceptible de l'engager et ne fonde plus de mouvement qui ferait école dans
l'histoire du lyrisme, que ce soit à l'Est ou à l'Ouest. [...] [Elle] réfléchit sur sa propre
histoire, sur son rapport au langage, à la tradition et se prend [...] très souvent elle-
même pour thème, tant ses fondements esthétiques et sa légitimité sur le plan
poétologique sont faibles et ne vont pas de soi ${ }^{1}$.

De fait, la réflexion sur la variété des solutions poétiques appliquées par les aînés à des contenus équivalents comme la relation au langage, le rapport à la tradition ou l'autoréférentialité semble davantage préoccuper la génération des années 80-90 que les audaces de l'attitude avant-gardiste. Par ailleurs, la pluralité et le flou des formules employées: Neue Unübersichtlichkeit ("une absence de perspectives claires à l'heure actuelle», Jürgen Habermas), Ende der Meta-Erzählungen («la fin du récit autoréférentiel », Jean-François Lyotard), temps du anything goes («tout est permis », Paul Feyerabend), reflètent les difficultés de la critique à caractériser une époque où tout est possible et permis, où aucune tendance, pour autant qu'elle en ait les moyens, ne revendique le privilège de dominer le champ littéraire. Le temps des grandes certitudes est définitivement révolu, «[...] nous vivons au siècle des reprises. Chaque nouvelle avant-garde ferait ressurgir les fantômes qu'elle désire conjurer ${ }^{2}$ », assure Jürgen Becker. Par conséquent, poursuit-il, la prédominance accordée à l'instant élégiaque, en cette atmosphère fin-de-siècle, conduirait à méconnaître l'ambivalence d'un moment de l'histoire littéraire qui commence par la rupture de la modernité et 
reste ouvert à tous les emboîtements possibles sur des modèles existants. Aussi le sentiment d'appartenir à une époque finissante s'accompagne-t-il, chez l'artiste, et avec la même valeur, de la conscience claire que les possibilités du discours poétique demeurent, sous une autre forme, non pas selon le mode avant-gardiste de l'innovation radicale, mais comme la tentative de nouer une relation productive avec une tradition dont on n'ignore cependant pas la décrépitude, faisant ainsi perdurer l'écriture. C'est en cela, selon Becker, qui en réfère à Joachim Sartorius, auteur de l'anthologie Atlas der neuen Poesie, citant lui-même Klaus Martens, que réside désormais la tâche du poète :

[...] Pour le poète né à une époque finissante, l'impératif n'est pas d'innover, mais de développer une technique qui utilise l'inévitable "érosion des procédés poétiques imaginés comme un moyen fondateur de l'identité $»^{3}$.

3 Rien de surprenant donc à ce que le poète Ulrich Schacht, né en 1951 et dont l'expression poétique se nourrit, pour l'essentiel, de l'expérience de la nature, se réclame principalement de l'héritage de Peter Huchel (1903-1981) et Johannes Bobrowski (1917-1965), dont les œuvres respectifs entretiennent des rapports ambigus avec la Naturlyrik d'avant-guerre, en une confrontation permanente avec la tradition magique, s'en faisant à la fois l'écho et le prolongement. "Sans Peter Huchel que j'ai encore personnellement connu et Johannes Bobrowski à la veuve duquel j'ai rendu visite à Berlin dès 1970, je serai un poète lyrique d'une tout autre nature ${ }^{4}$, déclare-t-il en 1995. Certes, sans pour autant succomber à la tentation biographique, il est clair qu'une communauté de destin l'unit à ces deux hommes contraints à l'exil, loin du paysage natal - la Marche de Brandebourg pour Huchel, la Lituanie et la mythique Sarmatie pour Bobrowski - qui fut à la source de leur lyrisme. Né, en effet, dans une prison de femmes de l'ex-RDA où sa mère purgeait une peine de dix ans pour avoir projeté de fuir à l'Ouest avec un officier russe, père de l'enfant, Schacht devra aussi quitter le Wismar de son enfance et souffrir des blessures infligées par l'histoire récente aux paysages de l'Allemagne du Nord et de la Baltique. Après avoir assisté à l'écrasement du Printemps de Prague, il entre délibérément dans l'opposition. Son inscription à l'Université protestante de Rostock, sa participation à Prague à la manifestation organisée sur la tombe de l'étudiant Jan Palach, son adhésion à la CDU, sont, dans le contexte de l'époque, des actes subversifs qui lui valent une condamnation et un emprisonnement en 1973. Il en sortira en 1976, au moment de la grande vague d'émigration qui, après l'exclusion de Biermann, saisit les poètes de l'ex-RDA, nés dans les années trente. Il se fixera à Hambourg, puis en Suède ${ }^{5}$. De là, des thèmes communs : le déracinement, la dépossession de ses valeurs et de sa terre natale, la perte d'une culture et la volonté désespérée de retrouver des repères disparus.

4 Toutefois, au niveau des enjeux historiques du discours poétique qui nous intéresse au premier chef, la filiation qu'il revendique replace son œuvre dans le débat sur la poésie de la nature qui, au temps de la partition, traverse, d'un côté comme de l'autre, la poésie allemande. Ainsi et de façon abusive, la critique a souvent assimilé, aussi bien l'art huchelien que le projet sarmate au modèle déjà contesté de la magie d'avantguerre, tel qu'on le trouve chez les Naturmagier comme Oskar Lœrke (1884-1941) et Wilhelm Lehmann (1882-1968). A tort, elle a considéré la poésie de ceux-là comme anachronique et purement restauratrice, estimant qu'elle marquait une régression dans l'histoire littéraire. Les années soixante, en revanche, ont vu s'affronter les héritiers conservateurs de la magie et de jeunes poètes contestataires comme Hans Magnus Enzensberger ou Peter Rühmkorf, désireux de se réorienter vers un certain type de poésie didactique issu du paradigme brechtien, cependant que, dans l'ex-RDA 
des années 70, la renaissance du Naturgedicht permettait à une nouvelle génération de prendre ses distances avec le moule fonctionnel du réalisme social. La production de Schacht, entre les années $1981^{6}$, date de son premier recueil et 1995 , pour ce que l'on connaît de l'œuvre en cette période, chevauche deux univers de l'histoire littéraire et politique de l'Allemagne. Elle s'alimente aux nombreux courants qui ponctuent, dans ce pays, l'évolution du lyrisme de la nature. C'est à double titre qu'elle tire sa légitimité des résidus d'une esthétique périmée. Cultivant à la fois le doute huchelien et le nouveau rêve d'unité bobrowskien, elle tente d'ériger son propre édifice, à l'instar de ses devanciers, conscients d'avoir derrière eux le monde du savoir et de l'harmonie encore célébré par la génération magique.

\section{I}

5 Le poème sarmate se définit comme un lieu d'expérimentation où l'écriture confronte la conscience aux fragments d'une histoire à recomposer, après les terribles destructions subies par le pays pendant l'invasion allemande. Seule une lecture attentive permet de déloger les vérités dissimulées dans une nature marquée du sceau de l'histoire. À l'évidence, Schacht, dénonçant la cruauté du système de défense estallemand, a retenu le geste de dévoilement à des fins cognitives, basé sur un mélange spécifique de réalisme et de magie. Comme Bobrowski qui énumère avec précision les éléments du paysage ( Wege fallen dir zu / und die Flüsse, Jura und Mitwa, / jung, aus Wäldern, und lehmschwer / Szeszupe. [...]», « Die Memel», SZ, 49-507), Schacht, dont les poèmes portent des noms géographiques ("Der Priwall», "Nordeney», "Der Culpiner See », « Pyrmonter Prospekt », «Dänischer August », « Mœn », « Wismar von See her ", «Vor Sredni », 79 $30^{\circ} \mathrm{n}$. Br.), insère son discours dans la réalité concrète : «[...] seule la dénomination exacte de ce qui constitue notre sol natal et qui en fait, par là, une perte fondamentale, est susceptible, au sens figuré, de le conserver, sans pour autant le remplacer, bien sûr. ${ }^{8}$ ", affirme-t-il. Il va de soi qu'il ne s'agit pas, au sens où l'entendait la magie conventionnelle, de transposer l'existence propre des éléments naturels dans le poème, mais de mettre en lumière l'interférence du monde sensible et $\mathrm{du}$ phénomène culturel, afin de montrer comment l'histoire humaine se lit symboliquement dans la nature. Pour cela, il emprunte à ses prédécesseurs deux procédés poétiques, à Huchel sa métaphore et à Bobrowski l'effet syntaxique de l'apokoinu, dans «Der Priwall», nouant ainsi le dialogue, sur le plan thématique et formel, avec des auteurs qui, face aux bouleversements politiques et à l'évolution de la pensée, durent développer des techniques d'expression propres à traduire un autre mode de relation au monde :

Die [Bojen] warnen die Menschen

und Schiffe gelb-eine

eitrige Narbe vom Strand ins Meer vom Meer zum

Strand [...] : Nur

Möwen und Krähen und losgelassene gefesselte Hunde

im Sandkorndickicht lauert

die Stille im Stahlkorndickicht

sensible Maschinen die schleudern

eiserne Dornen in jeden der hoffend

sich grenzenlos hingibt- 
Die Landschaft dahinter

ist lieblich und still.

(« Der Priwall », SS, 18)

6 En l'absence du lien, selon les leçons de Gottfried Benn, entre comparant et comparé, ce n'est pas tant la vision des bouées délimitant la frontière inter-allemande sur la Baltique comme des cicatrices purulentes, témoins d'une blessure qui ne guérit pas, que le contraste entre les composés "Sandkorndickicht / Stahlkorndickicht» qui, à la manière de Huchel, diabolise la nature. Le maintien d'une fonction référentielle au sein du langage métaphorique fait apparaître la duplicité des phénomènes réels qui contribue à la perception négative du paysage, agressé par l'histoire politique (cf. " kreuzotterndickicht ", " Das Zeichen ", PHGW I, 113 et l'opposition "Sternenreuse ", Die Sternenreuse / "Stacheldrahtreuse », «Ophelia », PHGW I, 83-84 et 1759). L'acier s'est subrepticement glissé à la place de l'élément naturel et les machines à tuer sont devenues la substance même du réel. L'image " eiserne Dornen » parachève l'ancrage du langage métaphorique dans la matérialité du paysage. Elle fait cohabiter nature et histoire dans le corps du texte et instaure une tension entre la face visible et la face cachée du monde qui déstabilise la perception.

7 Il apparaît, en outre, que "lauert» se rapporte aussi bien à "Stille » qu'à «Maschinen», ces instruments de mort avec lesquels l'homme a trahi et perverti l'innocence de la nature. L'ambiguïté ainsi entretenue reproduit l'opacité d'un monde où le sujet a perdu sa souveraineté. En sus, la remise en cause de la perception ordinaire par la destruction de l'image convenue maintient la fonction heuristique de cette dernière, fonction qui procède d'une intention démonstrative, présente dans le cycle sarmate et, à un degré moindre chez Huchel, et que Theo Mechtenberg relève chez Schacht: «[...] faire voler en éclats l'image idyllique du paysage et révéler la menace de mort ${ }^{10} »$. Le dépaysement provoqué agit en agent destructeur d'un autre mode d'appréhension du réel, celui de l'idylle, esquissée dans les deux dernières lignes. La perception simultanée du merveilleux et de l'idylle constitue une part de l'héritage qui relie Schacht à ses prédécesseurs, eux qui savaient mettre au sein de la plus petite unité, comme dans la structure des ensembles, un subtil dosage de valeurs antagonistes pour donner à voir une autre dimension.

8 À cette occasion, Schacht réaménage le modèle du poème-cadre employé par Huchel à ses débuts, aux fins de déconstruire la magie. À la représentation d'un passé exemplaire dans la première partie du poème, la plus importante, Huchel oppose, généralement, de manière abrupte, celle d'un présent déficitaire, énoncé seulement dans les deux derniers vers ("Am Beifußhang», PHGW I, 57) ou dans la strophe finale («Der Knabenteich », « Löwenzahn », PHGW I, 59-60 et 80-81). Parmi les trois groupes de vers qui composent « Slutuper Idyll » (TG, 45, Slutup, point de passage de la frontière interallemande aux abords de Lübeck), le premier, consacré à l'Ouest, et le dernier, à l'Est, enserrent une scène pastorale figurant par inversion la zone neutre. La zone de la mort (Todesstreifen) est devenue une sorte de biotope, un jardin d'Eden dans lequel s'ébattent en liberté des animaux de légende. La disposition graphique reconstruit ainsi un espace géopolitique où se lit le destin de l'Allemagne. Par le rapprochement de réalités antithétiques, Huchel met en lumière, contrairement à la Naturmagie qui vise plutôt à les neutraliser, les tensions qui subsistent entre le moi élocutoire et le monde naturel. Chez Schacht, la spatialisation paraît, presque à elle seule, donner une crédibilité à la vision et inviter le lecteur à un déchiffrement du réel. L'image plastique fait coïncider signifiant et signifié. Est-ce un retour au concept imitatif de l'art? 
L'écriture, chez Bobrowski, repose sur l'hypothèse d'une mémoire dissimulée dans l'expression même du paysage. La mémoire épouse les contours d'une nature qui associe le nom et la matière à une esthétique complexe du signe. S'il reprend, effectivement, le topos du langage de la nature, celle-ci ne s'avère pas toute disposée à divulguer son secret, au contraire de Eichendorff, par exemple, chez lequel le déchiffrement d'une vérité cryptée qui se cache dans les hiéroglyphes du monde sensible, paraît aisé. Les handicaps simulés de la nature (surdité, mutisme) font aussitôt prendre conscience d'une réalité qui se rétracte et disparaît à l'approche de l'individu (" [...] die Spur / Mehl hinter sich, / taub, ohne Rede », " Alter Hof in Häme », WZ, 69). Les hiéroglyphes, comme les objets auxquels ils renvoient, se replient sur leur secret et tout espoir d'interpréter les signes par l'intervention de la conscience directe doit être abandonné. Chez Schacht, l'expérience d'une nature qui ne se révèle pas, qui se défend, ouvre également la réflexion, non à une vérité supérieure, mais à une humanité sans repère ni mémoire solide :

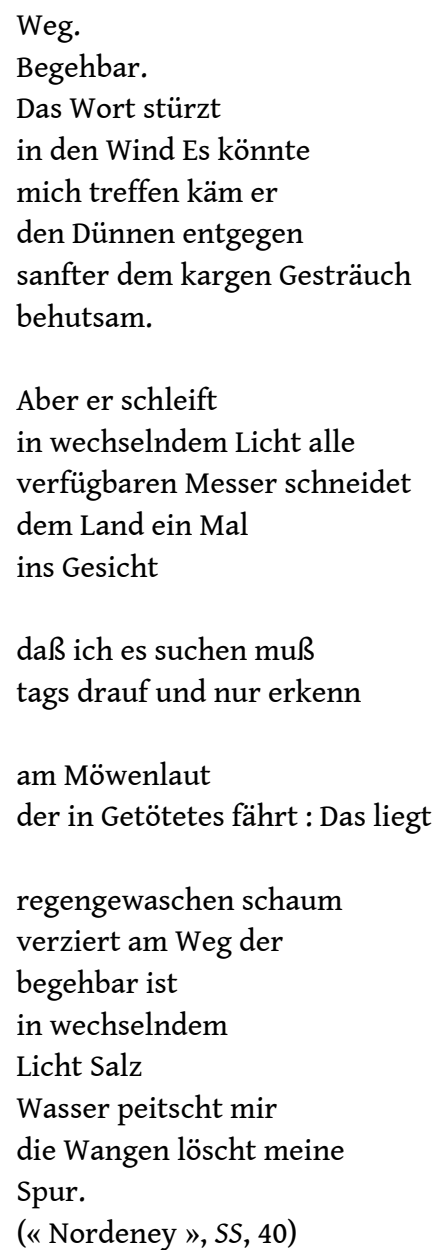

10 Ainsi qu'en témoigne le conditionnel « es könnte mich treffen [...]» (cf. «wär ich ein Nachtgesträuch », "Nachtweg », SZ, 43), le projet de rencontre demeure irréalisable, le rêve impossible de la symbiose sépare encore le sujet de l'objet de sa quête. Le contenu sous-jacent du message n'est, toutefois, pas simplement masqué par une symbolique codée dont le sens profond, chez Bobrowski, n'échappe que provisoirement à une magie 
qui s'applique à relever les traces hermétiques que la nature imprime en tous lieux. La nature de "Nordeney " se révèle d'une agressivité telle que le désir de reconnaissance, à l'exemple de Huchel, se double d'une incertitude sur le pouvoir référentiel du signe («Atmet noch schwach: Durch die Kehle des Schilfrohrs, / Der vereiste Fluß?», "Winterpsalm», PHGW I, 154-55). Le chant du monde qui s'exprime, de manière dérisoire, dans le cri des mouettes ne renvoie qu'à un univers mort. La disparition même du signe lavé par la pluie annihile toute chance de rédemption: «[...] löscht meine Spur» (cf. "War es das Zeichen? // Erstarrt / Im Schweigen des Schnees, / Schlief blind / das Kreuzotterndickicht", "Das Zeichen», PHGW I, 113-14). Semblablement, à l'heure de midi, instant privilégié de l'illumination poétique, le fiat créateur échappe à la subjectivité («[...] Gegen Mittag blendet / der Sand mich // nicht », « Dänischer August », SS, 43). Les pistes semblent tout à fait brouillées pour qui tente de rassembler les débris d'un langage éclaté ("Spuren einer Scherbensprache », selon le mot de Theo Mechtenberg ${ }^{11}$ ) qui, autrefois assurait l'unité du monde ( Licht / ins Meer schlägt / auf zerbricht: Scherben / Spur bis an den Rand », "Dänischer August ", SS, 43).

11 En même temps, le traitement thématique de l'oubli comporte une interrogation profonde sur le rapport de l'individu à ses souvenirs perdus et sur l'intégration possible dans un espace signifiant :

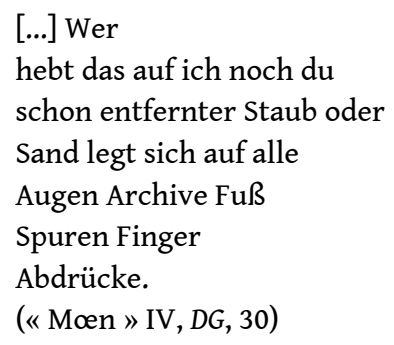

12 L'analogie avec l'ensevelissement des morts sur les yeux desquels on jette la poussière et le sable (cf. « Die mich einscharren / unter die Wurzeln, [...] », « Antwort », WZ, 46), la poussière elle même étant signe de l'éphémère, réduit à néant l'espoir d'une préfiguration visionnaire dans une épiphanie de la lumière. Non seulement les yeux, mais aussi les images du souvenir («Augen Archive», formation semblable à «Fuß Spuren » et « Finger Abdrücke ») que les yeux ont gardées ont disparu dans la poussière de l'oubli et de la mort.

"Was / wird aus einem Land / wenn sein Gedächtnis krank ist », ces vers de Guntram Vesper qui figurent en exergue au premier cycle de poèmes, «Umwarntes Gelände », du recueil Lanzen im Eis, montrent qu'à l'aube des années quatre-vingt-dix, la poésie de Schacht reste partiellement tributaire d'une esthétique sous l'égide de laquelle Helmut Arnzten classe l'œuvre : Alltagslyrik und Reminiszenz ${ }^{12}$. C'est que Vesper élabore aussi son discours sur la quête des traces du passé dont il s'agit de dénoncer les insuffisances, au même titre que celles du présent et l'on ne saurait être vraiment surpris que, dans la postface du recueil Frohburg ${ }^{13}$, il en réfère à l'un des deux maitres de Schacht, à Peter Huchel.

\section{III}

Dans le discours huchelien, les motifs de la soif (« Münze aus Bir el Abbas », PHGW I, 130-31), de la cécité (« Pe-Lo-Thien» dont les vers « Aus eisigen Wassern / tauchen die 
Tage auf, / störrisch und blind » figurent en exergue au $3^{e}$ cycle, « Dämmerungen », de "Lanzen im Eis », PHGW I, 219), de la surdité ("San Michele », PHGW I, 118 et Polybios, PHGW I, 119) marquent une rupture douloureuse entre la subjectivité et le monde qui mène aux lisières du silence. Il cultive la parole comme un ferment indispensable à l'écriture, de même que Bobrowski chez qui celle-là s'épuise à vouloir convoquer un passé inaccessible ("müder Mund», "Sprache», WZ, 38), mais dont la volonté d'échange et la ténacité finissent par neutraliser les handicaps. Le paysage et la parole, à statut égal, prennent alors indifféremment l'initiative d'une évocation qui ouvre, ainsi que le remarque Isabelle Durand-Henriot, l'extraordinaire mémoire du signe, jadis signifiant :

L'auteur parvient à faire du poème un foyer de convergences, un creuset où les principaux actants trouvent enfin un relatif équilibre. Désir du monde et désir de mémoire, manifestation objective et reconquête du moi s'y rejoignent en une unité féconde ${ }^{14}$.

Le rendez-vous de Schacht avec le Wismar d'autrefois ne déclenche pas le processus attendu de réappropriation de l'espace qui pourrait libérer les potentialités de la conscience :

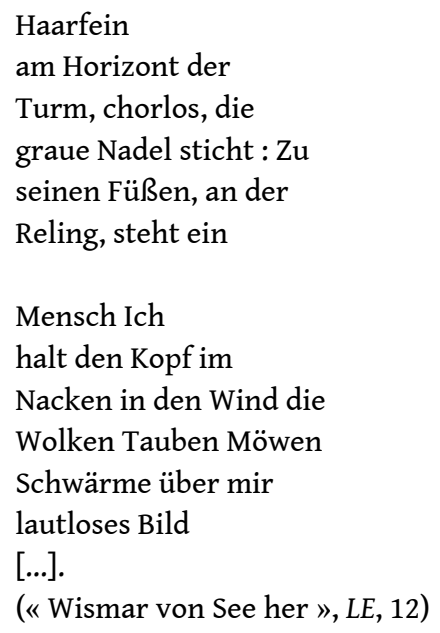

La localisation exacte de l'énonciateur, repoussée par l'enjambement à la ligne suivante, prolonge pour un temps la vision, aussitôt détruite. Puis le silence d'Orplid, l'île fantastique de la déesse Weyla dans le roman de Mörike, Maler Nolten (« Du bist // Orplid, / mein Land ! das / ferne schweigt [...] ») atteste de l'impuissance du discours à construire un espace fictif, habitable pour la subjectivité. Ce silence compromet toute promesse de synthèse dans l'utopie littéraire, telle qu'elle s'avère pourtant réalisable chez Bobrowski (« [...] Nun im Dunkel / halt ich dich fest », « Die Memel », Gedichte, 53).

À y regarder de plus près cependant, la séquence médiane où la subjectivité apparaît en force, constitue les prémices d'une évolution qui conduira l'écriture à une plus grande autonomie face à l'héritage. La proximité, sensuelle, avec la nature garantit de nouvelles perspectives («über mir ») et la juxtaposition d'unités de même nature au centre du poème («Wolken Tauben Möwen / Schwärme [...]») souligne l'adhésion du langage au réel. Ce qui compte désormais, ce n'est pas tant le rejet de l'idylle que l'évolution interne de l'individu et la reconnaissance progressive de ses repères. La révision de la subjectivité est, en ce cas, un mécanisme essentiel à la prise de conscience et, dans cette optique, le poème « Küste bei Fyr » s'emploie à dédoubler les perspectives par la confrontation de deux identités concurrentes, un moi premier, implicite, dont les 
facultés restreintes s'opposent à la lucidité d'un sujet second, actualisé dans des tournures impersonnelles :

Der durch den Kreide Bruch

steigt atemlos unbeschwert

Sanddorn und Distelgewöll

erreicht sieht

zwischen weißen Klippen :

Das Meer. Grün

bis zum Grund von großen

Vögeln samenkornklein

überflogen -

Der so das Meer sieht ist

allein. Und nicht traurig

Er ruft. Und wartet.

auf keinen.

(« Küste bei Fyr », DG, 31)

L'absence de recouvrement des instances, implicite et explicite, instaure une tension qui illustre la difficulté. La résistance au changement s'exprime dans le caractère, imprimé dans la forme, laborieux et pénible de l'ascension des falaises de craie. À l'exemple du locuteur qui escalade la roche, le lecteur doit, en effet, escalader les arêtes de la syntaxe, buter sur la fragmentation des composés, trébucher sur les enjambements et vaincre l'ambiguïté des articulations logiques pour, en l'absence de toute ponctuation, restituer la linéarité du discours. Il parvient, dans le même temps, au point de vue en surplomb et, par conséquent, à la connaissance. À lui seul, le texte oppose une résistance à la compréhension immédiate comme les épines et les chardons (le chardon, une pièce maîtresse dans l'inventaire poétique de Huchel !) qui freinent la progression du grimpeur, dans le mouvement d'élévation qui symbolise l'avènement du langage poétique à l'existence. Les effets syntaxiques prêtent au langage les caractéristiques matérielles de la nature. La parole poétique semble déléguer au paysage le pouvoir d'opérer une synthèse encore impossible au niveau de l'instance narrative.

L'accès au nouveau mode d'appréhension du réel est subordonné à l'autorité subjective du regard (Der $s^{15}$ das Meer sieht [...]) qui intègre puis élargit les perspectives de la séquence initiale. C'est par l'intensité du regard que se réalise, dans "Pyrmonter Prospekt », le mariage du réalisme et de la vision (« [...] aber nichts : rührt sich außer / all // meinen Augen [...] Forsythien : Blätter verlieren / den Halt : Ihr Fall übertönt / den Zug den ich: erwarte.", "Pyrmonter Prospekt», LE, 34). L'inversion de la perception habituelle, les arbustes ne perdent pas leurs feuilles, ce sont ces dernières qui perdent leur support, et le signe de ponctuation suffisent à opérer la jonction entre l'empirique et le visionnaire. Le visuel s'impose donc ici comme un outil privilégié qui sonde et enrichit l'espace. Ainsi se trouvent posées non seulement la question de la relation entre le moi et le monde, mais aussi celle de la relation entre le moi et une perception du monde qui puisse fonder une parole poétique. "L'assimilation de la chose regardée aide le moi à se saisir de son identité, mais [...] au moment où la chose est regardée, elle n'est rien d'autre que ce que le regard en fait ${ }^{16} »$, observe Françoise Lartillot. 
On mesure, en outre, l'autonomie formelle prise envers l'héritage. La grande économie des moyens mis en œuvre pour forcer la perception nouvelle des phénomènes et inviter à la spéculation du sens, échappe, bien évidemment, au caractère onirique de la métaphore huchelienne, «der blaue Fisch » ou « des Mondes Schnee» (le poisson bleu et la lune neigeuse, "Die Insel Aloe ", PHGW I, 22) qui associe le refus d'un réalisme primaire à la révision tout aussi critique des normes quotidiennes. Le procédé s'éloigne également des choix de Bobrowski dont la démarche investigatrice et conquérante, par le biais de l'invocation injonctive ( « [...] Lehr mich reden, Gras, / lehr mich tot sein und hören, lange, und reden, Stein, / lehr du mich bleiben, Wasser, [...]», «Ebene », STS, 59), en tant qu'acte verbal d'appropriation, contraste avec la passivité d'une instance subjective, soucieuse de ne pas soumettre le monde à une lecture abusive. C'est pourquoi l'ouverture sur une vision élargie, tendue entre histoire et utopie s'effectue davantage à l'insu du sujet que par l'action déformante et aliénante que celui-ci risquerait d'exercer simultanément sur le langage et sur le réel :
Als der Regen aufs
Meer fiel neben den
Bordstein vom Haus
die Wellen tätowierte
den Stein noch runder
werden ließ, widerfuhr
meinen Augen ein Bild
späterer Tage an denen
der Regen aufs Meer
fiel neben den Bordstein
vorm Haus nur die Nummer
hat sich geändert der
Name der Küste die
Richtung in die mich
der Wind treibt.
(« Wetter », Bericht, $L E, 85$ )

La magie ne naît pas d'une opposition univoque et brutale à la réalité objective, c'est bien plutôt le parallélisme établi entre les groupes prépositionnels, évoquant les phénomènes naturels à l'état brut («Regen », " Meer »), d'une part, et les témoignages culturels de l'espace (« Haus», «Bordstein »), d'autre part, qui favorisent l'intrusion du futur («Bild späterer Tage »). Le décrochement de la perspective n'a d'autre but que d'encourager l'ouverture sur un futur, même indéterminé, et de bâtir un univers aussi réaliste que prospectif où la subjectivité se fond. La vision ne découvre pas non plus un espace transcendant les frontières les plus reculées du monde sensible. Sans rompre avec le concret, elle crée une dimension transitoire avant que le présent ne vienne sceller l'unité du je, du monde et de l'histoire.

\section{IV}

Dès lors, c'est à travers l'expérience directe de la perte du pays natal que Schacht réussit, comme Bobrowski, à établir une relation positive au monde. La mise en œuvre de stratégies différenciées permet à l'un comme à l'autre, de dépasser le stade huchelien d'une nostalgie critique. Le motif du pays natal sert, pour ainsi dire, de déclencheur et de collecteur entre plusieurs univers poétiques. Or, c'est la découverte du monde arctique, au cours de trois voyages successifs, qui fournira véritablement à 
Schacht l'occasion d'une relecture critique et distanciée de ses modèles. La nature arctique a conservé ce degré de pureté nécessaire à l'instauration d'un rapport d'immédiateté, fondateur d'identité, occulté ailleurs non seulement par l'histoire politique, mais aussi par la réflexion sur la relation homme-nature et sur le langage qui jalonne l'histoire du lyrisme depuis la crise de la représentation.

Si les jeux d'enfants, sous forme d'exercices paramilitaires organisés le dimanche pour les jeunes pionniers, avaient désacralisé la forêt, ce haut lieu du locus amoenus et de l'inspiration poétique ( Der Wald / der uns nie verriet / der Wald / den wir verspielten », " Der Wald vor der Stadt », SS, 76), les couleurs de la nature arctique, en revanche, ne sont pas suspectes d'appartenance politique («Licht Bruch, ins Nichts / geschliffene Zeit. Die Farben / ohne Verdacht [...] », "Vor Sredni, 79³0 n. Br. », TEA, 12). Dans le giron de cette nature élémentaire, l'expérience conduit l'écriture à revisiter des modes d'expression antérieurs et à définir de nouveaux enjeux poétologiques :

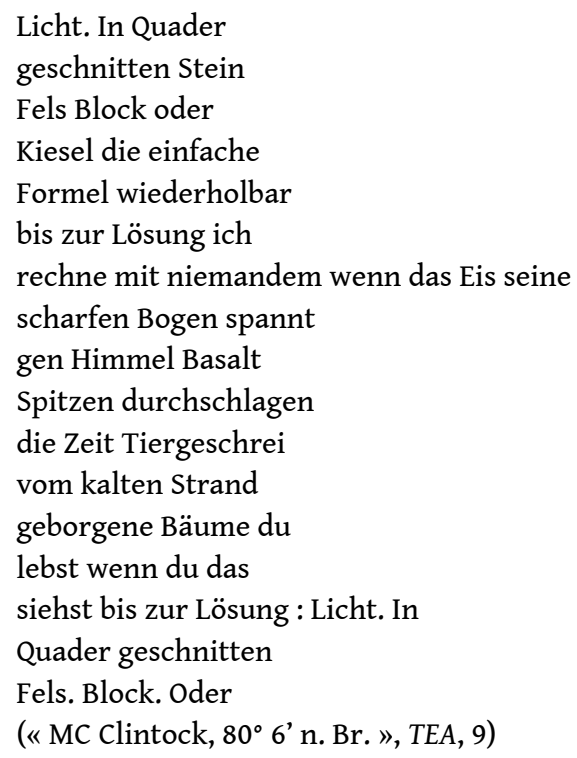

Assurément, Schacht reprend la technique ancienne de la nomination, un type de mise en relief que Bobrowski affectionne particulièrement. Au début du texte, le vocable mis en exergue s'impose sans article. Isolé, le nom semble constituer, à lui seul, le matériau original du paysage évoqué. Egalement présent en fin de poème, le procédé permet d'accroître la force des constellations nominales et d'illustrer le jaillissement du langage dans le monde, l'allusion à la lumière originelle n'est que trop évidente. Ainsi sont recensées les composantes primordiales du paysage : "Licht », "Stein », « Fels », «Block», «Eis", «Himmel », «Basalt», «Strand», «Baum». Est-ce à dire que l'initiative de la création revient au dire poétique qui exerce son pouvoir sur les êtres et les choses? «Die einfache Formel ", la nouvelle formule magique est d'une résolution simple, elle consiste à restituer aux phénomènes naturels leur entité primordiale, selon le mode de reconnaissance consacré quelques années auparavant face à la mer Morte ( « Wenn das Wort / Stein wird. Aufhebbar / und nicht zu bewegen [...] Wenn das Wort / Licht wird. Durchschaubar / und nicht zu durchdringen [...] », « Am Toten Meer », LE, 105). Il appartient au langage, non pas de créer son univers, qu'il soit partiellement déconnecté du réel comme la mythique Sarmatie ou totalement comme chez Günter Eich, (cf. "Akazien sind ohne Zeitbezug / Akazien sind soziologisch unerheblich / Akazien sind keine Akazien », «Zwischenbescheid für bedauernswerte Bäume », GEGW 
$\left.I, 174^{17}\right)$, mais de rendre la nature à elle même, purgée de ses repères antérieurs. Le Verbe redevient matière :

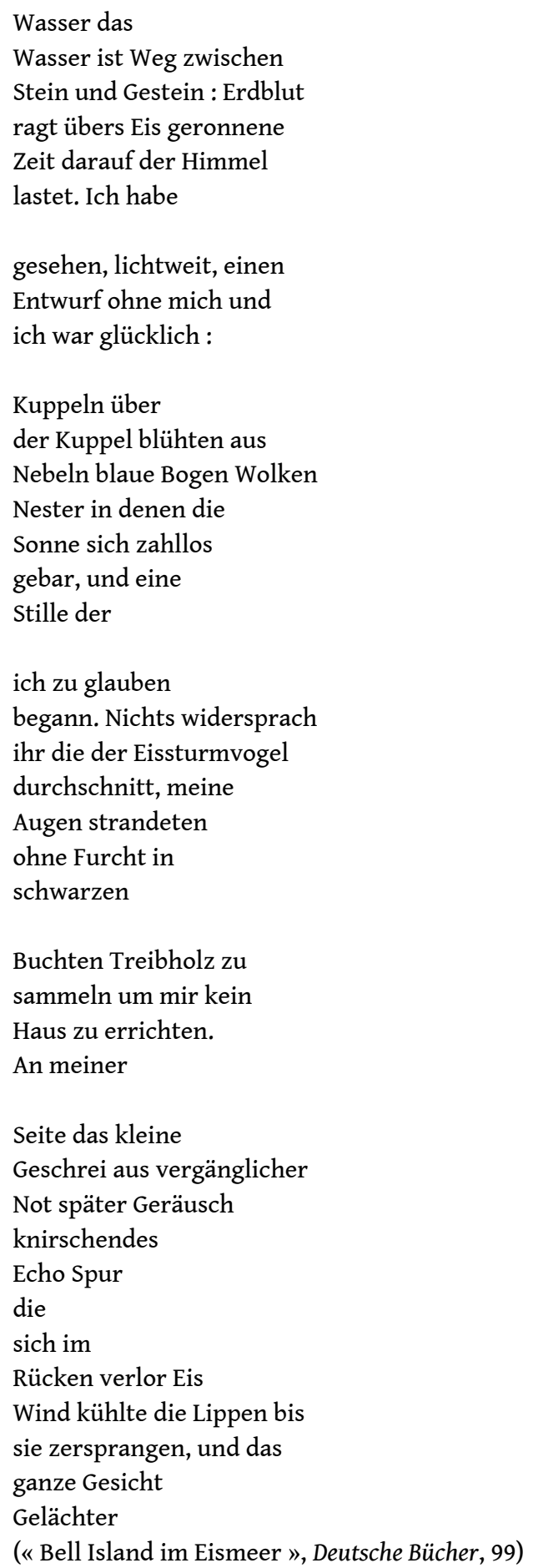

Le texte formule de nouvelles questions sur les ressources de la magie et de son héritage. La pratique de Schacht s'inscrit dans la lignée des discours poétiques identitaires que l'on pourrait caractériser par la manière dont ils réinvestissent l'écart constitutif de l'identité. Or, il apparait clairement que la relation univoque entre l'objet et sa dénomination a perdu, ici, son caractère problématique hérité de la crise du langage. À travers la réaffirmation de l'identité entre signifiant et signifié, le poème 
supprime d'un trait l'écart qui mène habituellement à la perte de réalité et à l'aliénation de la parole.

Inscrit dans la matière des choses, le silence, chez Huchel, est un dernier moyen de résistance, un acte de révolte passive (cf. «Traum im Tellereisen », PHGW I, 155-56). Par le refus du dialogue et le refus de la fusion, tels que les pratiquera Bobrowski dans son écriture polyphonique, le silence apparaît alors comme la dernière arme qui puisse préserver l'individualité d'une inévitable dissolution. À l'opposé, l'évocation schachtéenne construit ses motifs à partir de l'effacement élocutoire et physique de l'individu (« [...]du / lebst wenn du das / siehst bis zur Lösung [...]», « MC Clintock, 80 6 ' n. Br. », « [...] Entwurf ohne mich », « Bell Island im Eismeer »). « Il est rare que dans le lyrisme, un je se soit autant effacé aux fins d'accorder d'autant plus d'espace à une nature qui se manifeste en de puissants signes ${ }^{18}$ ", constate Theo Mechtenberg. Encore affublé d'une majuscule au début du poème, le pronom désignant le locuteur apparaît successivement inscrit avec une minuscule, puis sous forme de possessif (« mir »), enfin les caractéristiques de la personne ne sont plus introduites que par le défini ( im Rücken », "die Lippen », "das Gesicht»). L'instance élocutoire se retire du poème comme l'individu s'efface devant la nature. «Um mir kein / Haus zu errichten » est une allusion gœthéenne, reprise du Prométhée : « Doch lassen stehn, / Und meine Hütte ${ }^{19}$ " que Volker Braun réinvestira dans "Das Eigentum ${ }^{20}$ ». Au passage, l'auteur règle ses comptes avec l'instrumentalisation de la nature, prônée par le modèle du réalisme social.

La synecdoque n'atteste pas d'un langage menacé qui tenterait encore de s'imposer (cf. «Zerschmetterter Mund, / Du leuchtest die Finsternis an », « Polybios », PHGW I, 150) et là où la magie sarmate offre une vision dissociée du sujet et de l'objet, un rapprochement asymptotique qui ne confond jamais l'homme et le végétal, la symbiose avec le monde conduit, au contraire, à l'assimilation complète du sujet dans un cadre qui en transcende les insuffisances. La subjectivité ne décide d'ailleurs pas vraiment de se fondre dans la matière, elle y est, pour ainsi dire, poussée sous l'influence dominatrice de la nature («der Himmel lastet», « die Sonne [...] gebar», « Nichts widersprach / ihr \{der Stille\}»). Sous ce regard nouveau se déploient une temporalité et un espace ouverts à toutes les perspectives, ainsi qu'en témoigne l'absence de ponctuation en fin de poème.

28 Le fait de traverser l'archaïque, d'accepter, pour un temps, la confrontation avec l'inhabituel, voire l'effrayant, («[...] meine Augen strandeten / ohne Furcht [...]») permet d'aborder un domaine qui favorise une organisation modifiée de la parole poétique et de construire un lieu identitaire, habitable celui-là. Le néant est le point de départ d'une élaboration nouvelle et d'un univers différent («[...] Nichts tritt dir entgegen / Nichts hindert dich wortlos zu / ernten das sprachlos / machende / Wort ", « Leigh-Smith, $80^{\circ} 11^{\prime}$ n. Br. », Deutsche Bücher, 99).

Dans son ambition d'embrasser la totalité, le lyrisme polaire de Schacht renoue, semble-t-il, avec la conception romantique d'une lecture que ferait l'homme de la nature, portant la signature de Dieu dans les éléments qui la composent, car les phénomènes naturels sont décrits avec des notions empruntées au domaine des arts plastiques : «Entwurf », « Kuppeln über / der Kuppel » (cf. "Gravuren // auf endloser Seele [...] », "Vor Sredni, 79 30' n. Br. ", TEA, 12) et les six poèmes de "Svalbard », relient explicitement les deux champs sémantiques. Ils commencent par le même 
vocable «Stein » et contiennent aussi bien l'adjectif «violett» que, à l'exception de « Dänische Insel », le mot « Licht » :

Stein. Im Wasser treibt Eis. Vom

Frostrand bricht Licht Skulptur

um Skulptur : Gottes ewige

Vernissage. Alle Objekte im

Raum tragen das violette

Signet. [...]

[« Ny Alesund », Svalbard, 296)

«Vernissage » renvoie à l'art, "Signet » à la création littéraire et «violett » est une couleur qui entretient un rapport avec la transcendance. De l'art, les Romantiques espèrent la réparation de l'aliénation qu'ils ressentent entre l'homme et le monde. Il s'agit de créer par le monde fictif du poème le miroir dans lequel se reflète le monde idéal et de réconcilier ainsi l'idéal et la réalité pour accéder à l'harmonie perdue. Sauf que Schacht reconstitue l'unité non pas à partir d'un monde fictif, mais de l'expérience d'une réalité objective authentique. Avec le monde polaire, il ouvre, d'ailleurs, un domaine nouveau à l'expression lyrique.

«Comme si le bonheur résidait dans le fait que la pierre demeurât tout en haut : comme si le sens de l'écriture consistait à ne plus écrire! Par conséquent, la suite !21» Cette remarque, à la hussarde, de Jürgen Becker à propos du mythe de Sisyphe, convient à une génération qui développe un type de lecture ne supposant pas l'abandon d'une tradition, mais bien sa reprise distanciée. Pareille caractéristique introduit chez Schacht un balancement entre une consécration de la perte qui s'exprime dans la tonalité élégiaque du deuil et une tentative de restauration de la totalité perdue. Même si l'artiste recherche une conciliation utopique dont sa parole est le maître d'œuvre, la réflexion sur le discours identitaire préserve ses poèmes de toute notification d'archaïsme. Dans l'avant-dernier recueil, Die Treppe ins Meer, paru en juin 2006, la figure récurrente d'Hamlet dont le « je » aperçoit encore le château depuis le bac qui le reconduit sur la côte suédoise, offre matière à la réflexion poétique, non seulement au terme de l'histoire événementielle qui a profondément marqué l'évolution personnelle de Schacht, mais aussi d'une histoire des formes poétiques, face à laquelle il lui appartient de se définir :

[...] : Im untergehenden Licht

das kleine Schloß am Ende der großen

Geschichte ; Möglichkeitsformen, die mich

erreichen. [...]

(« Helsingborg, Wayne's Coffee », TM, 3)

On exige d'Hamlet qu'il recherche les derniers moyens d'expression qui puissent délivrer le poète moderne de son impuissance à trouver les mots, car le mélancolique dispose à la fois de la connaissance sur l'essence du monde et de la vision utopique. Il possède une conscience aiguë de la situation poétique du moment et si son silence signifie un retrait du monde, c'est justement dans l'écart que se développe l'utopie. Cependant, à la différence du modèle huchelien ( IM Kalmusge, Ruch dänischer Wiesen ", GW II, 257), il ne s'agit plus de sauvegarder ce qui peut encore l'être, la nuit novalisienne est, malgré quelque hésitation, pleine de frémissements prometteurs :

[...] nichts

ist zu hören als eigener 
Atem eigener Schritt eignes

Gespräch. Über Bäume vielleicht. Über

Pappeln die noch den sanftesten Wind

überführn : Luft Hauch Regen

Geräusch. Souffleuse Nacht, und ihr

großer Text aus dem

Dunkel dahinter.

( « In Kroken, Skäne. Idyllen II », TM, 31)

33

Le dernier recueil, Weißer Juli, paru tout aussitôt, contient un essai, «Die Wiederentdeckung der Geschichte der Sonne. Versuch über die Poesie der Natur und ihr Erscheinen in der Natur-Poesie ", qui vaut pour un art poétique et dont le message s'articule clairement sur la toile de fond de l'univers magique que Peter Huchel et Johannes Bobrowski, chacun avec sa vision du monde et ses performances originales, ont enrichi de leurs apports successifs. Schacht renoue ainsi en profondeur les fils d'une tradition éclatée par de vifs antagonismes aussi bien idéologiques que poétiques.

Toutefois un étiquetage trop strict et prématuré ne situerait que grossièrement le poète et l'enfermerait dans des a priori. L'œuvre exige qu'on lui laisse encore le temps de mûrir et c'est sans doute l'avis de son auteur puisqu'une partie significative de celui-ci est encore à peine tombée dans le domaine public.

\section{NOTES}

1. «Die Lyrik vertraut am Ende der 80er Jahre keiner verbindlichen Zielperspektive mehr und bildet keine epochemachenden Schulen, weder in der DDR noch im Westen. [...] [Sie] reflektiert ihre eigene Geschichte, ihr Verhältnis zur Sprache, zur Tradition und macht sich [...] häufig genug selbst zum Thema: so wenig verbindlich und so wenig verständlich ist ihre ästhetische Basis, ihre poetologische Legitimation ", in : Hermann Korte : Geschichte der deutschen Lyrik seit 1945, Stuttgart : Metzler, 1989, S. 206.

2. «[...] Wir leben in einem Jahrhundert der Reprisen. Jede neue Avantgarde riefe die Geister herbei, die sie überwinden will», Michael Braun « Nachwort», in : Michael Braun / Hans Thill (Hg.) : Punktzeit. Deutschsprachige Lyrik der achtziger Jahre, Heidelberg, 1987, S. 166. Zit. nach Andreas Wirthensohn : Annäherungen an einen vorläufigen Zusammenhang. Zum Werk Jürgen Beckers, Würzburg : Königshausen \& Neumann, 2000, S. 269.

3. «[...] für den spätgeborenen Dichter \{ist\} das dringlichste nicht eine poetische Innovation, sondern die Entwicklung einer Technik, die die unaufhaltsame "Erosion der imaginativen poetischen Methoden als ein Medium benutzt, in dem Identität gefunden werden kann" ", Jürgen Becker : «Laudatio auf John Ashberry », in : Jahrbuch der Bayerischen Akademie der schönen Künste, München, 1992, Bd. 6, S. 633.

4. «Ohne Peter Huchel, den ich noch persönlich kennengelernt habe, und Johannes Bobrowski, dessen Witwe ich schon 1970 in Berlin besuchte, würde ich anders sein als Lyriker. ", Hartmut Laufhütte: "Gespräch mit Ulrich Schacht», in: Deutsche Bücher. Forum für Literatur. Autorengespräch-Kritik-Interpretation, 1995/2, Bd. 25, S. 92.

5. À partir de 1990, Ulrich Schacht, auteur d'une poésie qui, en raison du passé, mêle l'expérience de la nature à la réflexion sur l'existence et sur les libertés opère une conversion étonnante : il 
s'affiche avec l'extrême-droite, écrit régulièrement dans Junge Freiheit dont les collaborateurs évoluent dans la zone trouble du conservatisme démocratique et du radicalisme. Avec Heino Schwilk, il publie Das neurechte Pamphlet. Die selbstbewußte Nation: "Anschwellender Bockgesang " und weitere Beiträge zu einer deutschen Debatte, puis, toujours avec Schwilk, Für eine Berliner Republik, contre l'euro et pour le renouveau de la République, il est candidat du groupe populiste «Bund freier Bürger » aux élections du parlement de Hambourg. Pour finir, il quitte l'Allemagne en 1998.

6. Ulrich Schacht :

Traumgefahr (=TG), Pfullingen : Neske, 1981.

Scherbenspur (=SS), Zürich : Ammann, 1983.

Dänische-Gedichte $(=D G)$, Hauzenberg : Edition Toni Pongratz, 1986.

Lanzen im Eis (=LE), Stuttgart : Deutsche Verlags-Anstalt, 1990.

Svalbard. Ein Zyklus, in : Günter Figal / Heino Schwilk (Hg.) : Magie der Heiterkeit. Ernst Jüngers zum Hundertsten, Stuttgart, 1995, S. 293-299.

Der Tod ist eine reine Erfindung der Augen. (= TEA).

Non publié, ce recueil se compose de cinq parties : «Die einfache Formel » qui comprend douze poèmes ayant pour thème la région arctique, dits : " poèmes polaires ", puis " Die bloße Hostie ", « Die falschen Farben », « Die Trauer ohne Grund » et enfin « Die deutsche Linie ».

Les poèmes polaires sont connus grâce au travail de Vera Dindoyal : 'Mystische Landschaft' oder 'Entwurf ohne mich'? Die Rolle der Natur in der Lyrik Ulrich Schachts, Berlin : Weidler Buchverlag, 2003, S. 213-242. Quelques autres ont été publiés dans des revues isolées et six d'entre-eux dans Svalbard. Ein Zyklus, in : A.a.O., S. 293-299.

Die Treppe ins Meer. Schweden-Gedichte (= TM), Hauzenberg : Edition Toni Pongratz, 2006.

Weißer Juli. (=wj), Hauzenberg : Edition Toni Pongratz, 2006.

7. Les œuvres de Johannes Bobrowski sont citées d'après les éditions suivantes :

Sarmatische Zeit (=SZ) / Schattenland Ströme (=STS)., Stuttgart : Deutsche Verlagsanstalt, 1961/62.

Wetterzeichen. (=WZ), Berlin : Klaus Wagenbach, 1967.

Gedichte (=Gedichte), Leipzig : Reclam-Verlag Leipzig, 1990.

8. «[...] nur das exakte Benennen dessen, was unseren heimatlichen Grund und damit den grundsätzlichen Verlust ausmacht, kann ihn - im geistigen Sinne - aufheben, ohne ihn - dies sei eingeräumt - zu ersetzen », Ulrich Schacht: Gewissen ist Macht. Notwendige Reden, Essays, Kritiken zur Literatur und Politik in Deutschland, München, Zürich, 1992, S. 29. Zit. nach: Vera Dindoyal, A.a.O., S. 159.

9. Les poèmes de Peter Huchel sont cités d'après : Axel Vieregg (Hg.) : Peter Huchel. Gesammelte Werke (=PHGW I), Frankfurt-am-Main : Suhrkamp, 1984, Bd.I.

10. "[...] das idyllische Landschaftsbild aufzusprengen und tödliche Bedrohung freizulegen », Theo Mechtenberg : "Nirgends ist Nähe so fern », in : Deutschland-Archiv, 1985, Bd. I, S. 91.

11. Ibid., S. 91.

12. Helmut Arntzen : "Alles geht? Beliebiegkeit und Form in der Lyrik der achtziger Jahre. Ein Vortrag ", in: Von Celan bis Grünbein. Zur Situation der deutschen Lyrik im ausgehenden zwanzigsten Jahrhundert, Germanica, 1997, Bd. 21, S. 20-21.

13. Cf. Hermann Korte, A.a.O., S. 200.

14. Isabelle Durand-Henriot: Magie et temporalité dans les poèmes sarmates de Johannes Bobrowski. La réception d'Oscar Lœrke et de Peter Huchel, Lille : Presses Universitaires du Septentrion, 2000, p. 200.

15. Souligné par nous.

16. Françoise Lartillot: Le Lieu commun du moi. Identité poétique dans l'œuvre d'Ernst Meister (1911-1979), Bern, Berlin, New-York, Paris, Wien, Peter Lang, 1998, p. 136.

17. Les poèmes de Günter Eich sont cités d'après : Axel Vieregg (Hg.): Günter Eich. Gesammelte Werke, Frankfurt-am-Main : Suhrkamp, 1991, Bd. I, S. 174. 
18. Theo Mechtenberg, in : Neue Zürcher Zeitung, $11 / 12$, 1984. Zit. nach : Vera Dindoyal, A.a.O., S. 234.

19. Wolfgang Gœthe : «Prometheus », in : Erich Trunz (Hg.) : Gœthes Werke, Hamburg : Christian Wegner, 1968, Gedichte und Epen, Bd. I, S. 44-45.

20. Volker Braun : «Das Eigentum », in : Marcel Reich-Ranicki (Hg.) : Deutsche Gedichte und ihre Interpretation, Frankfurt-am-Main : Insel, Bd. 10, S. 269.

21. «Als ob das Glück darin läge, daß der Stein endlich oben bliebe: als ob der Sinn des Schreibens der wäre, nicht mehr zu schreiben! Also weiter im Text.» Zit. nach : Andreas Wirthensohn, A.a.O., S. 274 .

\section{RÉSUMÉS}

Peu enclin à définir de nouvelles perspectives qui l'engageraient dans une posture avant-gardiste dont il désire éviter les excès, le lyrisme allemand contemporain paraît davantage préoccupé par son rapport à la tradition sur laquelle tous les emboîtements demeurent possibles. Aussi le lyrisme de la nature renoue-t-il, chez Schacht, avec l'art de deux poètes, Peter Huchel et Johannes Bobrowski, qui surent, à leur heure, développer de nouveaux procédés poétiques, aux fins d'adapter et de prolonger la magie d'avant-guerre. A l'instar de ses modèles, Schacht éprouve le rapport à la nature, fondateur de l'identité poétique, au travers de l'expérience douloureuse de la perte du pays natal dont le motif sert de connecteur interdiscursif. C'est par le regard nouveau, posé sur le monde, que la subjectivité parvient, au fil de l'évolution politique et culturelle du milieu où elle baigne, à dépasser aussi bien le stade huchelien d'une nostalgie critique que la stratégie de reconstruction bobrowskienne, par trop conquérante. La reconstitution provisoire de l'utopie ne se fait pas sans partager, semble-t-il, certains présupposés avec l'esthétique romantique et néo-romantique des prédécesseurs directs.

Anscheinend ist die zeitgenössische Lyrik wenig daran interessiert, neue verbindliche Zielperspektiven zu definieren, die sie zum avantgardistischen Radikalismus verpflichten würden und vor deren Exzesse sie sich nun schützen möchte. Dabei ist sie vielmehr mit ihrem Verhältnis zur Tradition beschäftigt, an die jede Artikulierung möglich ist. Schachts Naturlyrik knüpft an die Kunst zweier Naturdichter wie Peter Huchel und Johannes Bobrowski an, deren imaginierte poetische Methoden die herkömmliche magische Welt neugestalteten und bewahrten. Am Beispiel seiner Vorbilder prüft der Künstler durch die schmerzliche Erfahrung vom Verlust der Heimat - als intertextueller Konnex - die identitätsstiftende Relation zur Natur. Die Überwindung des kritischorientierten elegischen Gestus Huchels sowie diejenige der allzu dringlichen Eroberungsstrategie eines Bobrowski erfolgt schließlich durch neuartiges Sehen, durch das die vorläufige Rekonstruktion der Utopie wie bei den Vorläufern auf der Basis - so scheint es- eines romantischen und neo-romantischen, ästhetischen Denkens beruht.

\section{INDEX}

Mots-clés : RDA 
AUTEURS

MARYSE JACOB

Nice 